\title{
EVALUACIÓN DE LA FLEXIBILIZACIÓN LABORAL EN MÉXICO
}

\author{
Enrique Kato-Vidal ${ }^{\mathrm{a}}$
}

Fecha de recepción: 17 de enero de 2020. Fecha de aceptación: 20 de julio de 2020.

$$
\text { https://doi.org/10.22201/iiec.20078951e.2021.Especial.69555 }
$$

Resumen. Las políticas económicas requieren ser evaluadas para respaldar aciertos o
para revertir errores. En México se implementó una legislación que flexibilizó el mer-
cado laboral y ha pasado tiempo suficiente para evaluar qué efecto salarial tuvo. El
objetivo del artículo es estimar el impacto de la legislación laboral en la brecha salarial
entre trabajadores (permanentes y temporales) con contrato escrito y trabajadores sin
él. Para tal efecto, se empleó el método de diferencias en diferencias (DID, por sus siglas
en inglés) para detectar el impacto causal de la legislación laboral. Los resultados se in-
terpretan en una vertiente reconocida por Jaime Ros (Márquez y Ros, 1990; Ros, 2004
y 2019), relativa a una economía con excedente de trabajo y la necesidad de grandes
inversiones para superar las bajas tasas de crecimiento.

Palabras clave: diferencias salariales; reforma laboral; empleo; mercado de trabajo; estimación de diferencias en diferencias; contratos.

Clasificación JEL: E24; E26; J31; J38; 010.

\section{AN ASSEMENT OF LABOR FLEXIBILITY In MeXico}

\begin{abstract}
Economic policies must be subjected to scrutiny to allow successful policies to be reproduced and mistakes to be reversed. In Mexico, legislation that made the labor market more flexible was implemented and sufficient time has now passed to determine the effect this legislation has had on wages. The objective of this article is to estimate the impact of labor legislation on the wage gap between workers with written contracts and workers without written contracts, including both temporary and permanent workers. To this end, the difference-in-differences (DID) method was used to ascertain the causal impact of the labor legislation. Findings are interpreted within a framework described by Jaime Ros (Márquez and Ros, 1990; Ros, 2004 and 2019), one which is relevant to an economy with a labor surplus and the need for large investments to address low growth rates.
\end{abstract}

Key Words: wage differentials; labor reform; employment; labor market; difference in difference estimation; contracts.

\footnotetext{
${ }^{a}$ Universidad Autónoma de Querétaro, México. Correo electrónico: enriquekato@uaq.mx
} 
La teoría del desarrollo [...] vino a interesarse en un tipo particular de estado estable (trampas de bajo nivel) bastante ajeno a la teoría convencional de crecimiento.

Jaime Ros

\section{INTRODUCCIÓN}

Las diferencias salariales y de nivel de vida son un aspecto notable cuando se compara México con Estados Unidos y Canadá. Aunque la teoría predice que la integración económica (i.e. migración y comercio) promoverá la convergencia salarial, la realidad ha mostrado que esas diferencias permanecen (Gandolfi et al., 2017). El análisis de brechas o diferencias salariales también es un tema abierto hacia el interior de las economías nacionales. Por ejemplo, en Hernández Laos (2013) se revisa el debate respecto a si el mercado laboral mexicano está segmentado o integrado y en qué medida la flexibilización de la legislación permitiría cerrar la brecha productiva con la economía líder de Estados Unidos.

Desde la aprobación de la legislación laboral de 2012, en México, no se ha tenido en las revistas de investigación un debate sustantivo sobre el alcance y los resultados de la flexibilización del mercado laboral. Se puede decir que los análisis existentes se realizaron simultáneamente al proceso legislativo (Banco de México, 2013; Hernández Laos, 2013) o bien, han sido esfuerzos de evaluación poco sistemáticos. El presente artículo tiene como objetivo presentar la evaluación de la brecha salarial entre trabajadores con contrato y sin contrato escrito, previa y posterior a la legislación laboral mexicana de 2012. Para ello se adoptaron las categorías de presencia y ausencia de contrato por ser los términos en que se formuló la legislación (Banco de México, 2013).

En la perspectiva de Ros (2019), la distinción entre un empleo con contrato o sin él podría clasificarse ya sea como de informalidad primaria o de informalidad secundaria. La primera informalidad es más cercana a la teoría del desarrollo; mientras que la informalidad secundaria la describe como aquella que surge cuando las empresas eluden ilegalmente las contribuciones a la seguridad social, como resultado de distorsiones de política y de una fiscalización imperfecta.

Bajo este contexto, el presente artículo se circunscribe en la vertiente de informalidad primaria, dado que el grupo de comparación propuesto fue el 
amplio sector tradicional de trabajadores subordinados sin contrato. Se podría coincidir aún más con el concepto de informalidad primaria, por ejemplo, incluyendo en el análisis a trabajadores por cuenta propia, pero ello rebasa el objetivo principal.

Se eligieron a los salarios como variable de respuesta en el contexto de una legislación que buscó dar más contratos y ampliar el número de trabajadores del sector moderno ("formal"). En particular, la legislación laboral estuvo orientada a la eliminación de las barreras a la contratación y despidos, así como a modalidades flexibles de contratación. El resultado esperado era un aumento de la productividad al reasignar a trabajadores de baja productividad ( v.gr. sin contrato) a sectores modernos, como trabajadores permanentes o temporales (Banco de México, 2013). Dentro de todo el espectro de opciones evaluables, se eligió cuantificar qué ocurrió con la brecha salarial entre trabajadores con contrato y sin contrato, por considerar que es un indicador que contribuye a sintetizar la complejidad del mundo laboral.

Puesto que el objetivo del presente texto se limita a evaluar el efecto de la legislación, no se analizarán las premisas teóricas subyacentes. Se dirige al lector al artículo de Ros (2019) y de Ibarra y Ros (2019) para una discusión sobre la informalidad primaria y secundaria, la productividad sectorial y el impacto de la depreciación cambiaria y acumulación de capital por sectores.

Desde el punto de vista de Ros, la estrategia para aumentar el sector moderno de una economía con excedente de trabajadores en el sector tradicional debe consistir en elevar sustancialmente la tasa de inversiones para incrementar la intensidad de capital, sobre todo, en el sector tradicional. En opinión de Ros (2013a y 2013b), la flexibilidad del mercado laboral, y la eliminación de distorsiones microeconómicas, no pueden ser una fuente de crecimiento de largo plazo. Este planteamiento se basa en la interpretación de una economía como la mexicana conformada por una dualidad de sectores (i.e. tradicional y moderno), en lugar de la interpretación convencional de una economía madura à la Solow.

Para interpretar los resultados de la evolución salarial posterior a la legislación laboral se retomó un enfoque de dualidad de sectores. De esa manera, se pudo explicar el impacto negativo de la flexibilización laboral sobre el salario de trabajadores con contrato permanente y temporal. La estructura del artículo es la siguiente: después de la introducción, se presenta la revisión de la literatura, enseguida se exponen la metodología, los resultados, la prueba de robustez y, por último, se ofrecen las conclusiones. 


\section{REVISIÓN DE LA LITERATURA}

Ros (2013a y 2013b) señaló que cuando se ha estudiado el por qué los países pobres son pobres y qué tienen que hacer para escapar del subdesarrollo, las contribuciones de la teoría moderna del crecimiento, y de la nueva economía institucionalista, ignoran en gran medida la teoría clásica del desarrollo y sus aportaciones más recientes. La trascendencia de la teoría clásica se ilustra con el modelo de Lewis (1954) que por décadas fue retomado por Ros (Márquez y Ros, 1990; Ibarra y Ros, 2019). Una exposición detallada de ese modelo se publicó en el capítulo "Excedentes de trabajo" (Ros, 2004 y 2013b). Y resulta de interés dado que distingue las diferencias salariales entre una economía madura e industrializada y una economía en desarrollo (semiindustrializada) en donde coexiste un amplio sector tradicional que provee de trabajadores al sector moderno. En cambio, en una economía madura puede aplicarse el muy conocido modelo de Solow que relaciona la productividad y los salarios.

Para explicar una economía como la de México es insuficiente el modelo de Solow porque el sector moderno, que es relativamente intensivo en capital, no cuenta con el tamaño necesario para absorber a la mayoría de trabajadores del sector tradicional que opera con baja intensidad de capital y un bajo salario. Por ello, es útil recurrir al modelo de Lewis y explorar sus implicaciones para describir la evolución total de una economía con base en las interacciones entre los sectores tradicional y moderno. Por ejemplo, se puede expresar el producto por trabajador en el conjunto de la economía $(y=Y / L)$ como un promedio del producto por trabajador en los dos sectores $\left(w_{S}\right.$ y $y_{M}$ ) ponderando cada sector con su participación en el empleo.

$$
y=w_{S}\left(L_{S} / L\right)+y_{M}\left(L_{M} / L\right)=w_{S}+\left(y_{M}-w_{S}\right) L_{M} / L
$$

donde los subíndices $S$ y $M$ denotan al sector tradicional o de subsistencia y al sector moderno, respectivamente; y $L\left(=L_{S}+L_{M}\right)$ es el empleo total de la economía.

En un planteamiento como la ecuación 1, se asume que el sector moderno tiene una mayor productividad laboral que el sector tradicional y que es posible aumentar el ingreso per cápita de la economía a través de una reasignación de recursos, esto es, en la medida en que el sector moderno absorbe trabajadores del sector de subsistencia. Estos aumentos de ingreso terminan cuando todos los trabajadores tengan un empleo en el sector moderno. No resulta sencillo determinar si desaparecerá efectivamente en alguna fecha el sector tradicional o si ambos sectores coexistirán permanentemente. Un primer 
razonamiento suele ser que todas las economías se convertirán en maduras. Y ello consiste en la desaparición gradual del sector tradicional, y se calcula que este proceso podría durar décadas.

La posible extinción del sector tradicional se desafía en Márquez y Ros (1990) quienes califican a México, y a América Latina, como una experiencia particular de industrialización en la que persistió un gran sector tradicional (i.e. informal) a pesar del importante crecimiento experimentado entre 1950 y 1980. La alternativa, es decir, la posibilidad de la coexistencia sectorial tiene sustento teórico, tal que puede ocurrir en un equilibrio de largo plazo (Ros, 2004), siempre y cuando se cumplan algunas condiciones (v.gr. demanda elástica al precio en el sector tradicional y elasticidad de sustitución mayor que la unidad).

Independientemente de qué podría ocurrir en el largo plazo, en la actualidad existen implicaciones sobre las percepciones salariales dada la coexistencia de los sectores tradicional y moderno. Teóricamente se asume que el salario en el sector tradicional o de subsistencia $\left(w_{S}\right)$ se determina por su propio nivel de productividad (Ros, 2004; Lewis, 1954). Por su parte, el salario en el sector moderno $w_{M}$ depende de $w_{s}$ y de una prima salarial $f$. De esta manera, mientras los dos sectores coexistan se cumple que $w_{M}=f \cdot w_{s}$. La prima salarial se supone constante y de una magnitud suficiente para atraer trabajadores del sector tradicional al sector moderno. Una predicción importante del modelo es que un aumento de la inversión, es decir, de la intensidad del capital incrementa el empleo del sector moderno, pero no provoca un aumento salarial de $w_{M}$. Sólo cuando haya desaparecido el sector tradicional y, por tanto, todos los trabajadores estén contratados en el sector moderno $\left(L_{M} / L=1\right)$ las inversiones provocarán incrementos salariales.

En Márquez y Ros (1990) se identificaba que México aún estaba lejos de ser una economía madura dada la existencia de aproximadamente $40 \%$ de trabajadores en el sector tradicional. Los propios autores describen al sector con bajos niveles de productividad, sin barreras a la entrada o la salida, carente de estabilidad en el empleo, de seguridad social y de poder de negociación. En la actualidad, esas características siguen vigentes en México y también en economías de Europa del Sur (Hernández Laos, 2013; Gialis et al., 2017). En México, el gobierno federal aprobó en 2012 una legislación laboral para facilitar la modernización del sector tradicional (Banco de México, 2013). En síntesis, el balance del Banco de México fue que habría un sesgo de crecimiento hacia el sector moderno ( $v$ gr $r$ sector formal) al reducir las barreras para la contratación. Entre los cambios esperados se anticipaba un menor desempleo de jóvenes. 
Un punto de interés destacado por el Banco de México fue el horizonte en el que podrían evaluarse plenamente los efectos de la legislación laboral. Tomando la experiencia de las reformas en Alemania (2003-2005), el Banco de México (2013) asume que el nuevo estado estacionario podría alcanzarse en 2018, esto es, cinco ańos después de aprobados los cambios a la Ley Federal del Trabajo. No se conocen investigaciones sistemáticas que evalúen el alcance o resultados que tuvo la legislación laboral mexicana de 2012. Por lo que el presente artículo busca aportar elementos de análisis en esta materia.

Indagando en la literatura de Ros se puede identificar la simultaneidad entre el debate de la legislación laboral de 2012 y el libro Algunas tesis equivocadas sobre el estancamiento económico de México (Ros, 2013c). La obra fue escrita simultáneamente al debate del Pacto por México (Casar, 2019) que incluyó las modificaciones a la Ley del Trabajo. En aquel libro una conclusión de Ros (2013c) fue que, incluso eliminando todas las distorsiones microeconómicas, la mayoría de las empresas en aquel momento tradicionales (i.e. informales) seguirían siendo poco productivas dada la ausencia de nuevo capital (cfr. Perrotini, 2014). Para Casar (2019), los años transcurridos desde 2013 han validado la justa posición de Ros respecto a que persistiría el bajo crecimiento y la desigualdad.

Ros no hace ninguna mención del saldo que tuvo la legislación laboral de 2012, aunque tuvo oportunidades para hacerlo. Por ejemplo, en los cuestionamientos (Ros, 2019) que hizo a los planteamientos de Levy o también pudo haber mencionado el impacto de la legislación laboral cuando analizó el porcentaje de los ingresos que se paga como remuneración al trabajo. En cambio, Ibarra y Ros (2019) decidieron mantener el debate sin incursionar en los efectos de modificar las políticas laborales. Concluyendo que la evolución de la economía mexicana se debe a la lenta acumulación de capital y a la lenta expansión del sector moderno, en línea con muchos de sus resultados previos.

A diferencia del caso mexicano, el caso español es una experiencia más estudiada sobre los cambios a la legislación laboral, en particular, los cambios realizados en la década de 1990. En Cervini-Plá et al. (2014) se encontró que el efecto salarial asociado a la disminución de pagos por despidos fue prácticamente nulo; en cambio sí encontraron modificaciones salariales significativas provocadas por la disminución de impuestos. El nulo impacto de los pagos por despido es un aspecto interesante, ya que es un componente clave de la reciente legislación mexicana (Banco de México, 2013).

Conviene destacar que el sector tradicional español no juega un papel importante en el análisis del caso español. En uno de los artículos revisados, el estudio se centra en medir la variación salarial de un grupo particular de trabaja- 
dores, aquellos que cambiaron su estatus de desempleados a contratados de forma permanente (Cervini-Plá et al., 2014). En otro artículo, los tipos de trabajadores estudiados fueron los contratados temporalmente versus los que ostentan un contrato permanente (Davia y Hernanz, 2004).

De forma distintiva, una característica mexicana, y latinoamericana, es la presencia de un gran grupo de trabajadores sin contrato, lo cual vuelve relevante la inclusión de este grupo en una evaluación que busca observar los cambios de la legislación laboral.

\section{MeTOdología}

Para el presente análisis se eligió al salario como la variable de estudio. En general, se suele utilizarlo para identificar el efecto de las características de los trabajadores y también para estimar el impacto causado por cambios de legislación (Cervini-Plá et al., 2014; Gandolfi et al., 2017; Davia y Hernanz, 2004). En la literatura, un enfoque dominante para calcular el efecto salarial es el de diferencias en diferencias (DID, por sus siglas en inglés) (Imbens y Wooldridge, 2009; y Abadie, 2008). En ese sentido, se retomó el uso de DID para evaluar el impacto de la legislación laboral de México de 2012 y, en específico, se estimaron las diferencias salariales entre trabajadores con y sin contrato escrito, antes y después de implementada la legislación laboral.

La estimación se realizó utilizando datos de la Encuesta Nacional de Ocupación y Empleo (ENOE) (a partir de esta mención, Encuesta de Empleo) del Instituto Nacional de Estadística y Geografía (INEGI), ya que cuenta con representación nacional y proporciona elementos sobre la naturaleza y la calidad de los empleos. Para ello, el inegi utiliza un marco conceptual reconocido tanto por la Organización Internacional del Trabajo (оIт), como por la Organización para la Cooperación y el Desarrollo Económicos (OCDE) (INEGI, 2007). La diversidad de dimensiones que están disponibles en la encuesta permite controlar la heterogeneidad de condiciones laborales existentes en México. De la encuesta, el aspecto central que se retomó fue la identificación de la modalidad de relación laboral; en concreto, si el trabajador cuenta o no con un contrato escrito. El INEGi define el contrato laboral como la firma de un documento que regula la relación laboral con el patrón para el que laboran los trabajadores. Dada la ausencia o presencia de un contrato escrito se pudo subdividir la categoría de trabajadores subordinados entre aquellos con contrato ( $v . g r$ permanente o temporal) y aquellos sin contrato. 
A diferencia de los registros laborales del Instituto Mexicano del Seguro Social (IMss), la Encuesta de Empleo del INEGI recoge información de un universo más amplio; por ejemplo, incluye a trabajadores del sector público y a aquellos que laboran sin contrato en el sector privado. Dado que la Encuesta de Empleo contiene información similar, y adicional, a los datos del Imss, para realizar las estimaciones se empleó al INEGI como fuente principal de información y se usaron las cifras del IMss en una estimación adicional que se reporta como una prueba de robustez. Una ventaja al usar los datos del INEGI es la coherencia a lo largo del tiempo. En cambio, a partir de 2013 el número de empleos asegurados al IMss tuvo un repunte sostenido que es inconsistente con sus datos históricos y cuyas tasas de crecimiento son sospechosamente altas. En tal virtud, se privilegiaron los datos del INEGI, procurando el uso de datos sólidos tanto en fechas previas como posteriores a la legislación laboral de 2012.

La Ley Federal del Trabajo de México fue sustancialmente revisada durante 2012. La legislación laboral que resultó de esas revisiones fue publicada en el Diario Oficial de la Federación el 30 de noviembre de ese año. Para evaluar el impacto de los cambios a la ley laboral se eligió el periodo 2005-2017. Como año final se eligió 2017, dado que ya había transcurrido buena parte del tiempo estimado (cinco años) para observar el efecto total de la legislación (Banco de México, 2013). Se evitó usar 2018, ya que fue un año de elecciones presidenciales y suele haber un repunte económico que es indeseable para evaluar la política laboral. El punto de comparación se estableció en 2011 año previo a las negociaciones, con ello se buscó evitar que los datos de empleo estuvieran afectados por la incertidumbre asociada al proceso legislativo. De esa manera se establecieron dos intervalos:

a) 2011-2017: el periodo de vigencia de la ley, $y$

b) 2005-2011: el periodo base de comparación.

En los cuadros 1 y 2 se presentan los promedios de las variables para los tres años utilizados en las regresiones. 
Cuadro 1. México: trabajadores subordinados y remunerados (millones de personas) *

\begin{tabular}{|c|c|c|c|c|}
\hline & & 2005 & 2011 & 2017 \\
\hline \multirow[t]{2}{*}{ Sexo } & Hombre & 14.04 & 14.23 & 14.99 \\
\hline & Mujer & 7.50 & 7.93 & 8.16 \\
\hline \multirow{3}{*}{$\begin{array}{l}\text { Disponibilidad de } \\
\text { contrato escrito }\end{array}$} & Temporal & 1.99 & 2.10 & 2.14 \\
\hline & De base, planta o por tiempo indefinido & 9.73 & 10.15 & 10.38 \\
\hline & Sin contrato escrito & 9.65 & 10.20 & 10.44 \\
\hline \multirow{5}{*}{$\begin{array}{l}\text { Tamaño de las } \\
\text { unidades económicas }\end{array}$} & Micronegocios (con y sin establecimiento) & 5.23 & 5.93 & 5.83 \\
\hline & Pequeños establecimientos & 5.06 & 5.12 & 5.03 \\
\hline & Medianos establecimientos & 3.58 & 3.38 & 3.34 \\
\hline & Grandes establecimientos & 3.19 & 2.89 & 3.33 \\
\hline & Gobierno & 1.69 & 1.72 & 1.33 \\
\hline \multicolumn{2}{|c|}{ Localidades mayores a 100 mil habitantes } & 12.85 & 12.68 & 12.08 \\
\hline \multirow[t]{5}{*}{ Sector } & Construcción & 1.99 & 2.24 & 2.35 \\
\hline & Manufacturas & 4.54 & 4.39 & 4.81 \\
\hline & Comercio & 3.06 & 3.20 & 3.16 \\
\hline & Servicios & 10.13 & 10.79 & 10.42 \\
\hline & Agropecuario & 1.47 & 1.72 & 2.07 \\
\hline \multirow[t]{6}{*}{ Grupo de edad } & De 15 a 19 años & 1.83 & 1.65 & 1.43 \\
\hline & De 20 a 29 años & 6.64 & 6.83 & 6.56 \\
\hline & De 30 a 39 años & 6.06 & 6.06 & 6.10 \\
\hline & De 40 a 49 años & 4.26 & 4.72 & 5.10 \\
\hline & De 50 a 59 años & 1.98 & 2.47 & 2.88 \\
\hline & De 60 años y más & 0.69 & 0.86 & 1.05 \\
\hline \multirow[t]{5}{*}{ Escolaridad } & Primaria & 5.73 & 5.23 & 4.61 \\
\hline & Secundaria & 6.16 & 6.94 & 7.43 \\
\hline & Preparatoria & 3.26 & 4.27 & 5.18 \\
\hline & Carrera técnica & 1.52 & 1.18 & 0.09 \\
\hline & Profesional & 3.42 & 3.73 & 4.13 \\
\hline \multicolumn{2}{|c|}{ Empleadores y por cuenta propia } & 6.37 & 6.30 & 5.95 \\
\hline
\end{tabular}

Nota: * indica el promedio para trabajadores subordinados y remunerados que perciben más de un salario mínimo mensual. Fuente: elaboración propia con datos de la Encuesta Nacional de Empleo del INEGl y de la Comisión Nacional de Salarios Mínimos, México. 
Cuadro 2. México: ingreso mensual nominal (MXN)*

\begin{tabular}{llll}
\hline & 2005 & 2011 & 2017 \\
\hline Total & 4857 & 5614 & 6764 \\
$\begin{array}{l}\text { Disponibilidad de contrato escrito } \\
\text { Sin contrato }\end{array}$ & 3398 & 4064 & 5023 \\
Temporal & 4557 & 5554 & 6728 \\
De base, planta o por tiempo indefinido & 5859 & 6881 & 8142 \\
Grupo de edad & & & \\
20-29 años & 4104 & 4926 & 6039 \\
30-39 años & 5238 & 5923 & 7113 \\
40-49 años & 5707 & 6247 & 7348 \\
50-59 años & 5468 & 6339 & 7321 \\
Salario mínimo (MXN) & 1423.50 & 1819.53 & 2434.55 \\
\hline
\end{tabular}

Notas: * indica el promedio para trabajadores subordinados y remunerados que perciben más de un salario mínimo mensual. Fuente: elaboración propia con datos de la Encuesta Nacional de Empleo del INEGl y de la Comisión Nacional de Salarios Mínimos, México.

Se eligió como variable dependiente al logaritmo del salario mensual nominal. Se usa el salario nominal ante la falta de índices de precios específicos según edad o tipo de contrato de los trabajadores. Los resultados presentados con el salario nominal serían consistentes si la inflación ha sido similar para los grupos de trabajadores o si los trabajadores temporales han enfrentado una tasa de inflación mayor que los trabajadores permanentes. La estrategia realizada consistió en observar las diferencias salariales entre trabajadores con contrato escrito y sin contrato escrito en dos periodos: el primero de comparación o de control (2005-2011) y el segundo de análisis o tratamiento (2011-2017). Con esta información es posible evaluar el efecto de la legislación laboral en las diferencias salariales de trabajadores con y sin contrato. La estimación realizada se muestra en la ecuación 2.

$w_{i t}=\alpha_{0}+\alpha_{1} D_{t}+\alpha_{2} D_{i}+\beta^{\prime} \cdot\left(D_{i} \times D_{t}\right)+X^{\prime} \gamma+\epsilon_{i t}$

donde $w_{i t}$ es el salario promedio nominal del trabajador $i$ en el año $t, D_{i}$ es un vector de variables indicadoras el tipo de contrato laboral y $D_{t}$ es un vector 
de variables indicadoras para los años estudiados, el vector $X$ incluye variables explicativas como son: el sexo y el grupo de edad del trabajador, la escolaridad, el tamańo de la unidad económica, el sector de actividad y el tamaño de localidad, por último, $\epsilon_{i t}$ es el término aleatorio de la ecuación. Los $\alpha, \beta$ y $\gamma$ son parámetros que deben estimarse. Los coeficientes de interés de la ecuación 2 son los $\beta$ que representan los efectos de la legislación laboral; en particular, el efecto de la legislación sobre los salarios y las diferencias salariales estimados en 2017.

\section{RESULTADOS}

Como se planteó en la sección anterior, los resultados que se presentan buscan identificar los efectos salariales de la legislación laboral de 2012 en tres grupos de trabajadores: i) con contrato permanente o indefinido, ii) con contrato temporal y iii) sin contrato. La estimación usa la estrategia de DID para reportar una diferencia entre antes y después de la legislación laboral, así como una segunda diferencia entre trabajadores con contrato y sin contrato. Las estimaciones se realizaron por separado para dos grupos de edad: $i$ ) jóvenes y adultos jóvenes (20-39 años) y ii) adultos (40-59 años), encontrándose impactos negativos en cada grupo de edad.

El efecto de la legislación sobre los salarios se midió por los coeficientes $\beta$ de la interacción $\left(D_{i} \times D_{t}\right)$. En el cuadro 3 se muestra un resumen de los coeficientes estimados. Se halló un impacto negativo para trabajadores de todos los grupos de edad, tanto para los contratados de forma temporal como de forma permanente. El grupo etario con el mayor impacto negativo fue en el rango entre 40 y 59 años (véase figura 1c) y el tipo de contratación más afectado fue para trabajadores temporales. Después de la legislación laboral, el ingreso de trabajadores temporales se equiparó al de trabajadores sin contrato, cuando en años previos era de entre 2 y $3 \%$ más alto (véase figura 1a).

Usando los valores estimados del cuadro 3, el salario de trabajadores permanentes crece a una tasa relativamente constante de $3 \%$ anual, tanto para trabajadores jóvenes (20-39 años) como para adultos (40-59 años). En términos reales, esa tasa de variación salarial es negativa $(-1 \%)$ dado que la inflación registró una tasa de $4 \%$ promedio entre 2005 y 2017 . En términos generales, el ingreso de trabajadores sin contrato $(v \cdot g r$. sector tradicional) creció más rápido que el salario de trabajadores con contrato. Por ello, al paso de los años disminuyen las brechas salariales reportadas en la figura 1 .

Desde la perspectiva teórica, la prima salarial (o diferencia salarial) únicamente aumenta con el propósito de atraer a más trabajadores sin contrato ha- 
Cuadro 3. Efectos de la legislación laboral en los salarios

\begin{tabular}{|c|c|c|c|c|c|c|}
\hline \multirow[b]{2}{*}{ Variable } & \multicolumn{2}{|c|}{ Total } & \multicolumn{2}{|c|}{ 20-39 años } & \multicolumn{2}{|c|}{ 40-59 años } \\
\hline & Coeficiente & $t$ & Coef. & $t$ & Coef. & $t$ \\
\hline Contrato temporal & 0.021 & 3.90 & 0.020 & 3.01 & 0.034 & 2.98 \\
\hline Contrato de base & 0.156 & 44.07 & 0.147 & 31.85 & 0.163 & 24.50 \\
\hline Año 2005 & -0.215 & -70.90 & -0.221 & -55.35 & -0.195 & -31.60 \\
\hline Año 2017 & 0.205 & 69.13 & 0.201 & 50.33 & 0.215 & 37.70 \\
\hline$(\text { Temporal })^{\star}(2017)$ & -0.147 & -2.05 & 0.010 & 1.16 & -0.072 & -4.79 \\
\hline$(\text { Base })^{\star}(2017)$ & -0.024 & -5.70 & -0.012 & -2.11 & -0.051 & -6.73 \\
\hline Observaciones & \multicolumn{2}{|c|}{256135} & \multicolumn{2}{|c|}{145503} & \multicolumn{2}{|c|}{82724} \\
\hline
\end{tabular}

Notas: los coeficientes en negritas cuantifican el efecto de la legislación laboral. Estimación de mínimos cuadrados ordinarios. El grupo de control fue el de trabajadores sin contrato escrito. Año de comparación (2011). La estimación completa se puede consultar en el Anexo 1.

Fuente: elaboración propia con datos de la Encuesta Nacional de Empleo del INEGI, México.

cia el sector moderno en donde se ofrecen contratos permanentes. En México, la prima salarial ha disminuido, esto es, el sector moderno no parece haber tenido escasez de trabajadores, incluso ofreciendo salarios convergentes con el sector sin contratos (véase figura 1). Desde antes de la legislación laboral de 2012 comenzó a reducirse la prima salarial, pero la entrada en vigor de la legislación prolongó aún más la tendencia negativa y su impacto alcanzó de forma notable a los no jóvenes (40-59 años) y a la remuneración de los contratos temporales (véanse cuadro 3 y figuras 1a y 1c).

En concreto, previo a la legislación laboral, los trabajadores permanentes no jóvenes (40-59 años) tenían un salario entre 16 y $18 \%$ mayor que un trabajador sin contrato (2011 y 2005, respectivamente); para 2017 esa diferencia salarial se redujo a $11.2 \%$ (véase figura 1c). Por su parte, el impacto negativo al salario nominal de trabajadores temporales hizo que desapareciera la escasa brecha salarial que existía con trabajadores sin contrato en el grupo de trabajadores con 40-59 años. Esa brecha, previa a la legislación laboral fue de 3.5\%; el impacto de la legislación se estimó en $-7 \%$ (véase cuadro 3); de tal forma que, en 2017, un trabajador temporal percibía un salario menor (-4\%) que el ingreso salarial de un trabajador sin contrato (véase figura 1c). Este resultado puede ser polémico, por ello conviene citar el hallazgo de Davia y Hernanz (2004) quienes explican que trabajadores con dos modalidades diferentes de contrato tienen perfiles socioprofesionales muy distintos, pero si llegaran a coincidir, la modalidad menos ventajosa parece remunerar mejor al trabajador. 
Figura 1. México. Brecha salarial predicha (porcentaje respecto al ingreso laboral de trabajadores sin contrato)

a) Todas las edades

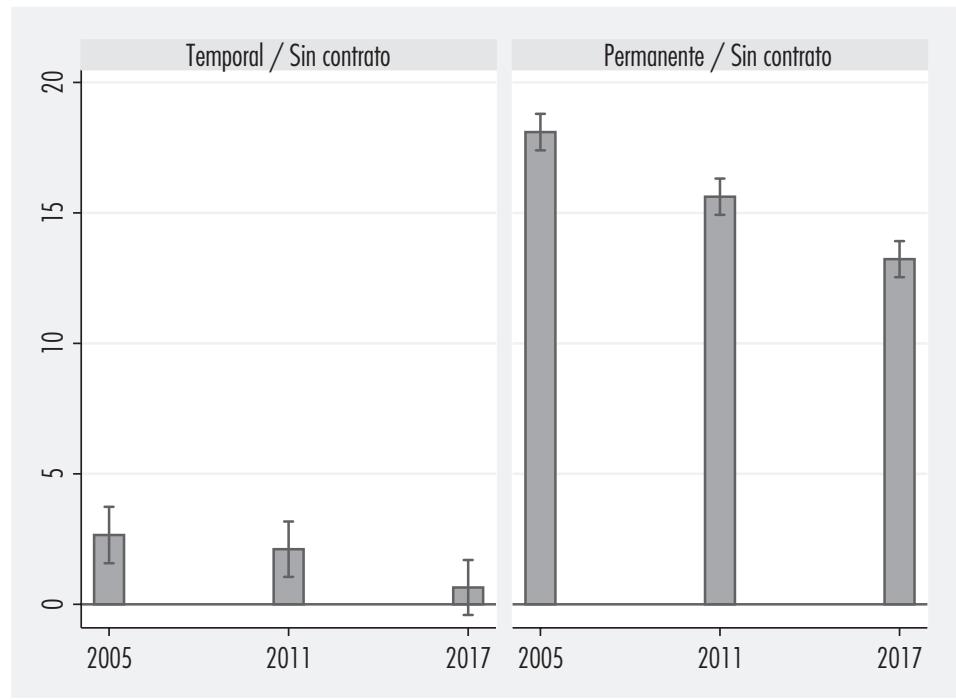

b) 20 a 39 años

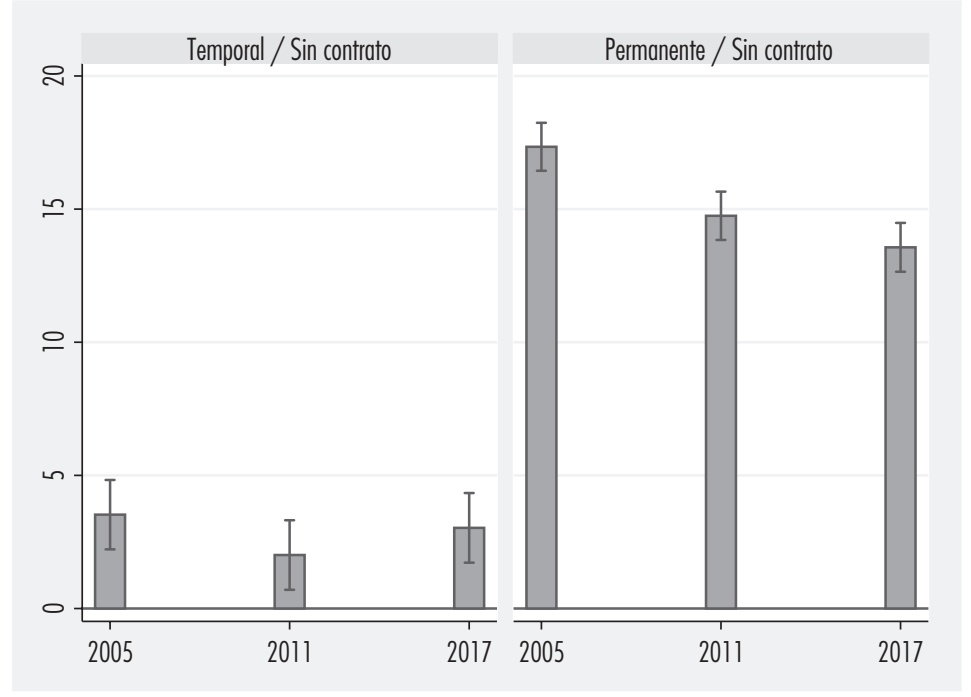

Continúa 
Figura 1. México. Brecha salarial predicha (porcentaje respecto al ingreso laboral de trabajadores sin contrato) (continuación)

c) 40 a 59 años

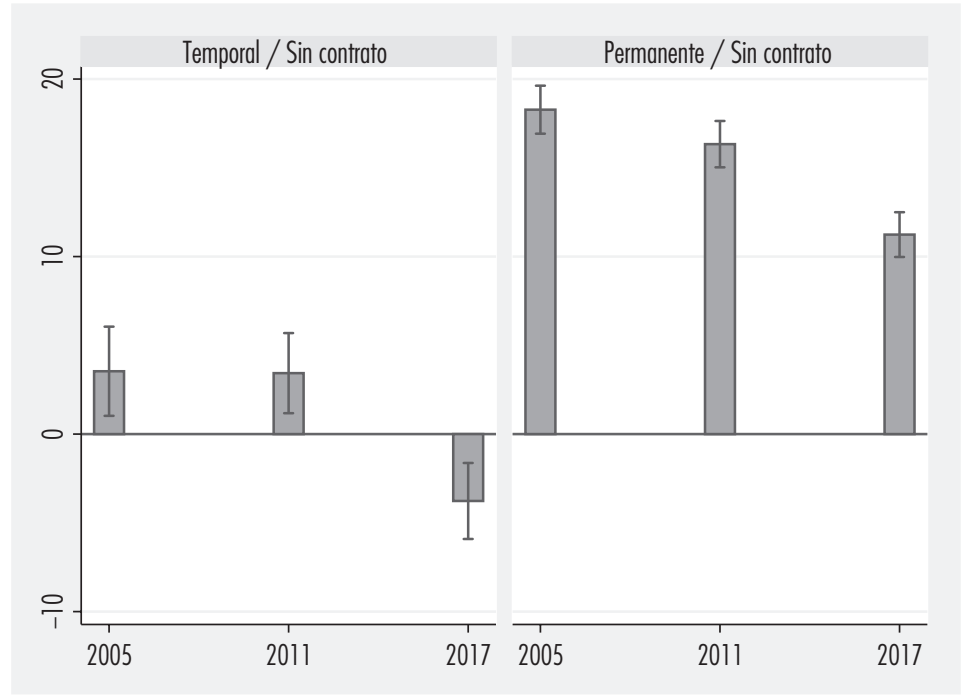

Nota: en cada barra se muestra un intervalo de confianza al 95\%. El efecto de la legislación se observa en 2017. Los años 2005 y 2011 muestran la situación previa a la legislación laboral. El grupo de 40 a 59 años fue el más afectado. Fuente: elaboración propia con los valores que predice la estimación del cuadro 3.

En comparación al impacto negativo que tuvo la legislación laboral en trabajadores de 40 a 59 años, la legislación tuvo un impacto diferente en trabajadores jóvenes (20-39 años). Por una parte, no se registró un impacto estadísticamente significativo sobre el salario de trabajadores temporales; $\mathrm{y}$, por otra parte, fue reducido el impacto negativo sobre el salario de trabajadores permanentes jóvenes (-0.012) versus en adultos (-0.051). En conjunto, estos resultados indican que a diferencia del grupo de más edad (40-59 años), en los jóvenes (20-39 años) se requiere mantener una mayor brecha salarial para atraer a trabajadores sin contrato al sector moderno que ofrece contratos escritos tanto permanentes como temporales.

Para contextualizar mejor los resultados presentados, en la siguiente sección se hará una prueba adicional usando una base de datos diferente al INEGI. Para ello, conviene ofrecer información adicional sobre las diferencias entre la muestra de trabajadores del INEGI y los registros de trabajadores del IMSs. Una primera diferencia es el promedio de edad capturado por el INEGI que es entre dos y tres años menor al del Imss. Así, en 2011, el trabajador representativo 
del IMss promedió 38.6 años y el trabajador en la Encuesta de Empleo fue de 35.5 años. Esta diferencia surge naturalmente porque al inicio de la vida laboral es muy frecuente que los trabajadores no reciban ninguna seguridad social.

Por otra parte, en los datos del INEGI, además de la subdeclaración de ingresos laborales y del problema de no respuesta a la encuesta, existe una subestimación de trabajadores con contrato permanente. Por ejemplo, en 2011, el INEGI estimó 10 millones de trabajadores permanentes y, ese mismo año, el IMss reportó 13 millones de trabajadores permanentes (véanse cuadro $1 \mathrm{y}$ Anexo 2, respectivamente). Si bien, los datos del INEGI tienen esta enorme desventaja, también tienen como beneficio excepcional el poder hacer comparaciones entre el grupo de trabajadores sin contrato y el de trabajadores con contratos temporal o permanente, lo cual no puede hacerse con los registros administrativos del IMss.

\section{PRUEBA DE ROBUSTEZ}

Como ya se expuso existen ventajas y desventajas al elegir cualquier base de datos para realizar las estimaciones. Bajo esta perspectiva, para corroborar la evidencia del INEGI, se ofreció como contraste una estimación con los registros del IMss. Y una crítica que podría hacerse a las estimaciones presentadas en la sección anterior radica en los posibles sesgos o inconsistencias internas de los datos del inegr. Para mejorar la calidad de los datos muestrales, se pudo aplicar un procedimiento de imputación de salarios. Otra posible solución consistió en usar otra fuente de información como la Encuesta Nacional de Ingreso y Gasto de los Hogares (ENIGH, INEGI) que también recolecta datos sobre los ingresos laborales en función de los contratos temporales o indefinidos. De entre las posibles opciones, se eligió la siguiente: hacer una prueba de robustez usando los registros administrativos del IMss.

A diferencia de las encuestas del INEGI, los registros administrativos del IMss sobre empleo, salario y tipo de contratación provienen directamente de las empresas. Esta característica contrasta en forma importante con los datos salariales del INEGI, que se obtienen al preguntar al trabajador o a un informante en su hogar. Por tanto, los registros del IMSs permiten corroborar la evidencia de la sección anterior. La estimación de corroboración se reporta en forma breve en el cuadro 4 (y en forma completa en el Anexo 3). Las variables usadas en la estimación fueron las mismas, la única excepción fue la exclusión de la escolaridad de los trabajadores, que no reporta el IMss. 
Cuadro 4. Prueba de robustez: efectos de la legislación laboral en los salarios de trabajadores registrados a la seguridad social

\begin{tabular}{|c|c|c|c|c|c|c|}
\hline \multirow[b]{2}{*}{ Variable } & \multicolumn{2}{|c|}{ Total } & \multicolumn{2}{|c|}{ 20-39 años } & \multicolumn{2}{|c|}{ 40-59 años } \\
\hline & Coeficiente & $t$ & Coeficiente & $t$ & Coeficiente & $t$ \\
\hline Contrato permanente & 0.054 & 5.92 & 0.016 & 2.14 & 0.103 & 7.88 \\
\hline Año 2005 & -0.346 & -41.62 & -0.363 & -45.78 & -0.56 & -35.13 \\
\hline Año 2017 & 0.308 & 27.79 & 0.312 & 30.15 & 0.300 & 23.70 \\
\hline$(\text { Base })^{\star}(2005)$ & 0.073 & 7.44 & 0.111 & 12.24 & 0.058 & 4.94 \\
\hline$(\text { Base })^{\star}(2017)$ & -0.039 & -5.51 & -0.047 & -7.96 & -0.028 & -2.97 \\
\hline Observaciones & \multicolumn{2}{|c|}{10603996} & \multicolumn{2}{|c|}{5410595} & \multicolumn{2}{|c|}{4240018} \\
\hline
\end{tabular}

Notas: los coeficientes en negritas cuantifican el efecto de la legislación laboral. Estimación de mínimos cuadrados ordinarios. El grupo de control fue el de trabajadores temporales (eventuales). Año de comparación (2011). La estimación completa se puede consultar en el Anexo 4.

Fuente: elaboración propia con datos del IMSS.

Al usar los datos del IMss, nuevamente se halló un efecto negativo y significativo de la legislación laboral, tal y como se reportó con los datos del INEGI. En términos generales, los hallazgos reportados son consistentes. Para identificar las diferencias (véanse cuadros 3 y 4) se expone lo siguiente: a) el efecto de la legislación laboral es más negativo entre el grupo de trabajadores con seguridad social (-0.039, datos del IMss) en comparación al total de trabajadores con y sin seguridad social (-0.024, datos del INEGI), y $b$ ) el impacto salarial negativo es mayor entre los más jóvenes (20-39 años) con seguridad social (-0.047, datos del IMSs versus -0.012, datos del INEGI). Estas diferencias podrían deberse a los problemas de subestimación y subdeclaración que tiene la Encuesta de Empleo. En todo caso, se sugiere interpretar las estimaciones como evidencia indicativa de un fenómeno laboral adverso y no como parámetros precisos de una realidad social.

Por último, faltaría discutir el porqué se afirma que la estimación presentada, ya sea en el cuadro 3 o en el cuadro 4, captura el efecto de la legislación laboral y no algún otro efecto diferente. Las estimaciones reportadas provienen de la ecuación 2 descrita en la metodología. Se puede observar que se tomaron varias precauciones para tratar de aislar o identificar el efecto de la legislación. Además de las diferencias por edad y sexo, entre las variables de control, se incluyó una tendencia para capturar los aumentos salariales previos 
a la legislación laboral (2005-2011) y otra para años posteriores (2011-2017). Estas tendencias fueron muy significativas. Los aumentos salariales posteriores a la legislación laboral (2011-2017) fueron de menor cuantía, respecto a años previos. Esta desaceleración es consistente con las menores inversiones de la etapa poslegislación (véase figura 2), lo cual brinda una cierta garantía sobre la autenticidad del parámetro de interés: el efecto de la legislación laboral.

Otros cuestionamientos que se consideraron al hacer la estimación fueron las diversas intensidades de capital que hay entre los distintos tamaños de empresa y entre los distintos sectores. Para ello, se incluyeron una serie de indicadores que estimaran el efecto salarial que aportan los distintos sectores y tamaños de empresa. En los anexos 1 y 4 puede revisarse que pagan mayores salarios las industrias más intensivas en capital y las empresas de mayor tamaño. Estas variables de control contribuyen a identificar más fehacientemente el efecto de la legislación laboral al aislar los impactos sectoriales y de intensidad de capital.

\section{LA INVERSIÓN Y LA PRODUCTIVIDAD}

Para complementar el análisis de los efectos de la legislación laboral mexicana de 2012, se ofrece la evolución que ha tenido la acumulación de capital y la productividad laboral. Ambas variables son clave para comprender las diferencias salariales. En la revisión de la literatura se destaca que la tasa de inversión permite la creación de empleo en el sector moderno de la economía. Pero la inversión de capital no es efectiva para lograr aumentos salariales cuando coexiste un amplio sector tradicional que provee trabajadores en forma relativamente elástica al sector moderno. En este contexto, Ros (2015) establece la imperante necesidad de inversiones que se requieren en economías como la mexicana para romper el bajo nivel de crecimiento registrado por tres décadas.

En la figura 2 puede observarse como en años de crisis (1995 y 2009-2010) se registra disminución en la tasa de inversión. En contraste, en los años posteriores a la legislación laboral (2012 y 2018) se tiene una caída sistemática de la tasa de inversión, algo que no se había observado en México en un entorno no recesivo. La figura 2 no es una prueba de causalidad entre la legislación laboral y la caída de la inversión, pero sí aporta elementos para comprender que la legislación laboral ha ocurrido en un entorno adverso con tasas inferiores al promedio histórico (16\% del PIB, 1988-2018) y una evolución negativa de la inversión. Esta tendencia es contraria a la deseable y necesaria. De hecho, desde hace años, ya se anticipaba que el esfuerzo de inversión requerido para 
Figura 2. México: tasa de inversión, previa y posterior a la legislación laboral

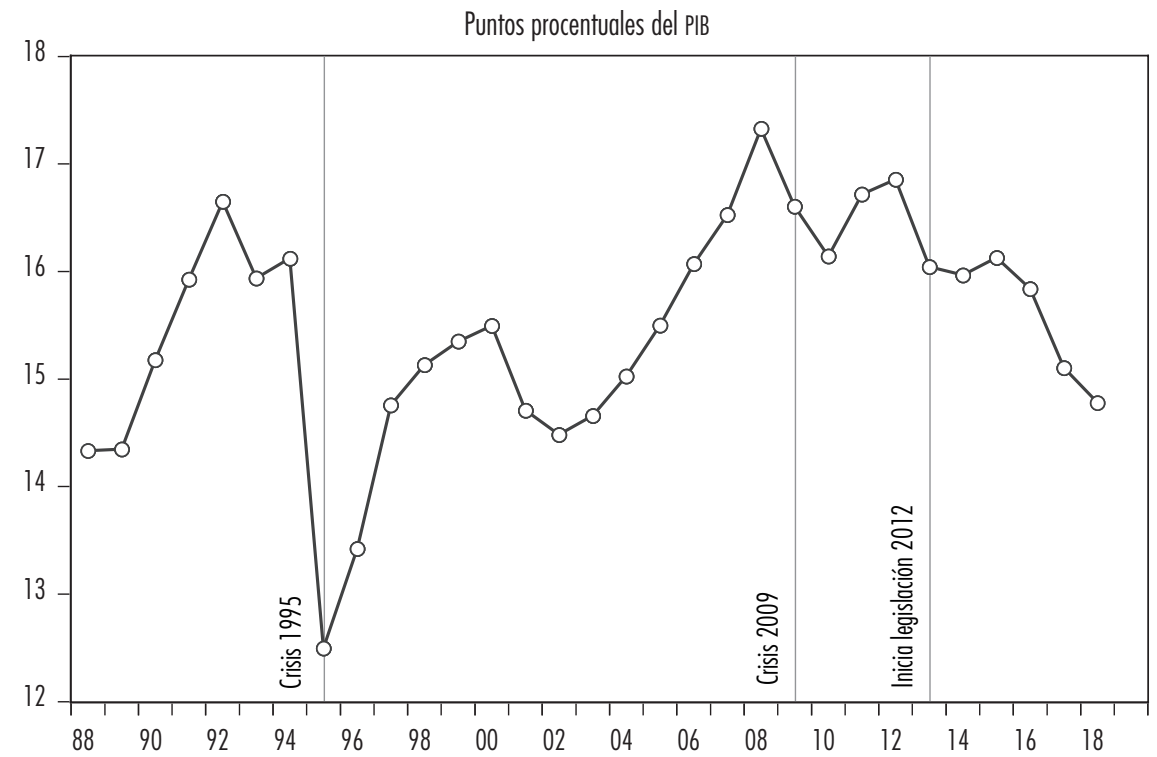

Fuente: elaboración propia con datos del Sistema de Cuentas Nacionales del INEGI, México. Cifras del periodo1988-2002 (año base 1993) y de 2003-2018 (año base 2013).

la modernización del sector tradicional tendría que ser mucho mayor que en el pasado cuando las actividades modernas evolucionaban gradualmente (Márquez y Ros, 1990).

Si bien la inversión ha tenido una tendencia negativa, la productividad laboral ha mantenido una tendencia positiva desde 2013. En la figura 3 se muestra la evolución de la productividad laboral. Conceptualmente, un aumento de la productividad se puede lograr reasignando trabajadores del sector tradicional con baja productividad hacia el sector moderno, el de mayor productividad, tal y como se planteó en la ecuación 1 . Se queda como una posibilidad sin comprobar que la reasignación sectorial puede ser la causa del repunte de la productividad en México en los años posteriores a la legislación laboral de 2012. En el cuadro 1 se pueden consultar las cifras de trabajadores según modalidad de contrato y puede corroborarse que, a pesar del menor número de empleos creados, hubo un pequeño sesgo a favor de los empleos con contrato escrito. 
Figura 3. México: productividad laboral, previa y posterior a la legislación laboral (escala logarítmica)

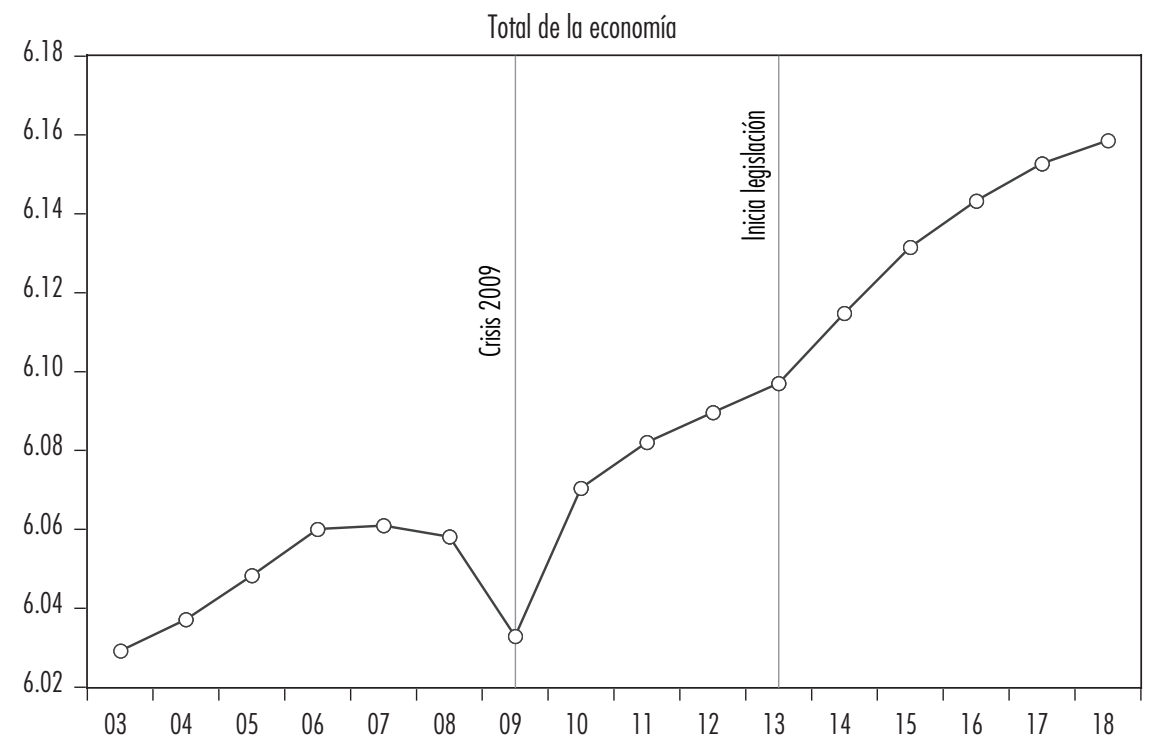

Nota: PIB por puesto de trabajo ocupado remunerado dependiente de la razón social (miles de pesos a precios de mercado, $2013=100)$.

Fuente: elaboración propia con datos de la Cuentas de Bienes y Servicios del Sistema de Cuentas Nacionales del INEGI, México.

\section{CONCLUSIONES}

El objetivo del artículo fue realizar una evaluación de las consecuencias de la legislación laboral mexicana de 2012 en la brecha salarial entre trabajadores con contrato y sin contrato escrito. Las estimaciones realizadas muestran que hubo un impacto salarial negativo de trabajadores de todas las edades (jóvenes y no jóvenes), el cual se observó con la disminución de la prima salarial entre trabajadores con y sin contrato, es decir, entre los sectores moderno y tradicional.

Las estimaciones permitieron identificar que la legislación laboral de 2012 prolongó y amplió los impactos salariales negativos que iniciaron varios años antes. Específicamente, antes de la legislación, ya se había registrado una reducción de la prima salarial entre trabajadores jóvenes (temporales y permanentes) respecto a trabajadores sin contrato. En 2017, al final del periodo de estudio, también se encontró una menor prima salarial entre trabajadores jóvenes permanentes y eventuales con seguridad social. Se destaca que los 
impactos sobrepasaron al grupo de trabajadores jóvenes. El mayor de los impactos se estimó en trabajadores de mayor edad (40-59 años) sin importar si laboraban bajo la modalidad temporal o permanente.

En general, los efectos atribuibles a la legislación laboral son consistentes con un modelo de economía dual à la Lewis. Aún con bajas tasas de inversión y con una menor prima salarial, el sector moderno logró aumentar marginalmente el número de trabajadores con contrato. En el fondo subsiste un sector tradicional (sin contratos) de proporción similar al sector moderno. Así, sólo algunos trabajadores se beneficiaron con contratos laborales. Por su parte, la mayoría de trabajadores del sector moderno tuvieron una menor prima salarial respecto a trabajadores sin contrato. Finalmente, sigue coexistiendo un enorme sector tradicional que mantiene reducido el salario promedio de la economía.

La contribución de este artículo es evaluar la legislación laboral, que fue una de las varias iniciativas económicas aprobada al inicio del sexenio 20132018. Estas iniciativas han sido poco examinadas en la literatura económica, a pesar de la polémica que crearon y del bajo desempeño que tuvo la economía mexicana durante ese periodo. Por otra parte, el análisis contribuye al método heredado por Ros ( $)$, descrito como: la necesaria renuncia a la explicación o modelo único de desarrollo y, por ello, la renuncia a recetas únicas para lograrlo (Casar, 2019); en consecuencia, antes de pronunciarse por un esquema particular de política económica, siempre debe examinarse la realidad a través de la evidencia empírica

Para aumentar la confiabilidad de los resultados se usaron dos fuentes de información diferentes. En la medida de lo posible, se buscó validar los resultados provenientes de la Encuesta de Empleo del INEGI que tiene problemas de subestimación del ingreso laboral, una alta tasa de no respuesta y un sesgo desconocido en la conformación de la muestra según el estrato socioeconómico. La validación se realizó reestimando los coeficientes con la base de datos de trabajadores registrados en el IMss. Se encontró coincidencia entre ambos resultados. Más adelante, el análisis se podría robustecer comparando similitudes y diferencias de trabajadores temporales registrados ante IMss y los que reporta la Encuesta de Empleo del INEGI.

Dado que el objetivo del artículo fue evaluar el impacto de una legislación no se profundizó en cuáles pudieran ser los canales específicos de la legislación laboral. En futuras investigaciones se podrían analizar esos canales, entre los cuales quizá estaría la intermediación de las empresas de subcontratación y las escasas inversiones e innovación del sector moderno de la economía. Por último, puede señalarse que una política procrecimiento apropiada al contexto 
mexicano debería basarse en un amplio programa de inversiones orientado a buscar una mayor intensidad de capital; dado que cuando existe excedente de trabajo en el sector tradicional, entonces las políticas que flexibilizan el mercado laboral no promueven crecimiento económico.

\section{ANEXO}

Cuadro A1. Estimación completa de los efectos de la legislación laboral en los salarios

\begin{tabular}{|c|c|c|c|c|c|c|}
\hline \multirow[b]{2}{*}{ Variable } & \multicolumn{2}{|c|}{ Total } & \multicolumn{2}{|c|}{ 20-39 años } & \multicolumn{2}{|c|}{ 40-59 años } \\
\hline & Coeficiente & $t$ & Coef. & $t$ & Coef. & $t$ \\
\hline Contrato temporal & 0.021 & 3.90 & 0.020 & 3.01 & 0.034 & 2.98 \\
\hline Contrato permanente & 0.156 & 44.07 & 0.147 & 31.85 & 0.163 & 24.52 \\
\hline Año 2005 & -0.215 & -70.85 & -0.221 & -55.35 & -0.195 & -31.55 \\
\hline Año 2017 & 0.205 & 69.13 & 0.201 & 50.33 & 0.215 & 37.66 \\
\hline$($ Temporal $) \star(2017)$ & -0.015 & -2.05 & 0.010 & 1.16 & -0.072 & -4.79 \\
\hline$(\text { Base })^{\star}(2017)$ & -0.024 & -5.70 & -0.012 & -2.11 & -0.051 & -6.73 \\
\hline$($ Temporal $) \star(2005)$ & 0.005 & 0.74 & 0.015 & 1.72 & 0.001 & 0.06 \\
\hline$(\text { Base })^{\star}(2005)$ & 0.025 & 5.80 & 0.026 & 4.64 & 0.019 & 2.41 \\
\hline Hombre & 0.176 & 92.16 & 0.172 & 69.95 & 0.204 & 57.31 \\
\hline Primaria & -0.155 & -54.95 & -0.114 & -30.63 & -0.232 & -42.88 \\
\hline Secundaria & -0.089 & -36.04 & -0.073 & -24.09 & -0.154 & -29.32 \\
\hline Carrera técnica & 0.071 & 17.29 & 0.072 & 13.24 & 0.046 & 6.37 \\
\hline Construcción & 0.236 & 57.53 & 0.221 & 42.02 & 0.262 & 32.59 \\
\hline Manufacturas & 0.019 & 6.27 & 0.012 & 3.11 & 0.033 & 5.43 \\
\hline Servicios & 0.091 & 33.06 & 0.090 & 25.61 & 0.105 & 18.83 \\
\hline Agropecuario & -0.059 & -8.90 & -0.073 & -8.53 & -0.093 & -7.35 \\
\hline $\begin{array}{l}\text { Micronegocios sin } \\
\text { establecimiento }\end{array}$ & -0.109 & -27.78 & -0.111 & -21.62 & -0.124 & -16.42 \\
\hline $\begin{array}{l}\text { Micronegocios con } \\
\text { establecimiento }\end{array}$ & -0.072 & -23.26 & -0.076 & -19.00 & -0.069 & -11.15 \\
\hline Medianos establecimientos & 0.048 & 16.82 & 0.044 & 11.86 & 0.062 & 11.74 \\
\hline Grandes establecimientos & 0.102 & 32.76 & 0.079 & 19.58 & 0.149 & 26.90 \\
\hline Gobierno & 0.094 & 25.51 & 0.099 & 20.09 & 0.080 & 13.11 \\
\hline
\end{tabular}


Cuadro A1. Estimación completa de los efectos de la legislación laboral en los salarios (continuación)

\begin{tabular}{|c|c|c|c|c|c|c|}
\hline \multirow[b]{2}{*}{ Variable } & \multicolumn{2}{|c|}{ Total } & \multicolumn{2}{|c|}{ 20-39 años } & \multicolumn{2}{|c|}{ 40-59 años } \\
\hline & Coeficiente & $t$ & Coef. & $t$ & Coef. & $t$ \\
\hline $\begin{array}{l}\text { Localidades mayores a } 100 \\
\text { mil habitantes }\end{array}$ & 0.082 & 45.66 & 0.092 & 39.47 & 0.076 & 22.60 \\
\hline Observaciones & \multicolumn{2}{|c|}{256135} & \multicolumn{2}{|c|}{145503} & \multicolumn{2}{|c|}{82724} \\
\hline De 15 a 19 años & -0.219 & -62.11 & & & & \\
\hline De 20 a 29 años & -0.124 & -55.96 & & & & \\
\hline De 40 a 49 años & 0.038 & 15.96 & & & & \\
\hline De 50 a 59 años & 0.038 & 12.74 & & & & \\
\hline De 60 años y más & -0.037 & -7.98 & & & & \\
\hline
\end{tabular}

Notas: estimación de mínimos cuadrados ordinarios. El grupo de control fue el de trabajadores sin contrato escrito. Año de comparación (2011). Los coeficientes en negritas cuantifican el efecto de la legislación laboral. Las estimaciones incluyen un término constante que no se reporta. Categorías de referencia: Sexo (mujer), Escolaridad (preparatoria), Sector (comercio), Tamaño de unidad económica (pequeños establecimientos). Tamaño de localidad (menores a 100 mil habitantes). Edad (3039 años).

Fuente: elaboración propia con datos de la Encuesta Nacional de Empleo del INEGI, México.

Cuadro A2. México: trabajadores asegurados al IMSS con salario (millones de personas)

\begin{tabular}{llccc}
\hline & & 2005 & 2011 & 2017 \\
\hline Sexo & Hombre & 8.09 & 9.36 & 11.90 \\
& Mujer & 4.32 & 5.34 & 7.03 \\
Tipo de contratación & Eventual (temporal) & 1.31 & 1.98 & 2.69 \\
& Permanente & 11.10 & 12.73 & 16.29 \\
& Un asegurado & 0.20 & 0.21 & 0.25 \\
Rango de tamaño del registro & Entre 2 y 5 asegurados & 0.90 & 0.93 & 1.10 \\
patronal & Entre 6 y 50 & 2.97 & 3.38 & 4.09 \\
& Entre 51 y 250 & 2.96 & 3.63 & 4.56 \\
& Entre 251 y 500 & 1.36 & 1.73 & 2.23 \\
& Entre 501 y 1 000 & 1.29 & 1.54 & 2.13 \\
& Más de 1 000 & 2.71 & 3.29 & 4.63 \\
Trabajadores en principales zonas metropolitanas & 5.27 & 6.30 & 8.03 \\
(CDMX, Estado de México, Jalisco y Nuevo León) & & &
\end{tabular}




\begin{tabular}{llccc}
\hline & 2005 & 2011 & 2017 \\
\hline Sector & Agropecuario & 0.26 & 0.34 & 0.51 \\
& Extractivas & 0.07 & 0.11 & 0.13 \\
& Transformación & 3.77 & 3.90 & 5.19 \\
Construcción & 1.00 & 1.19 & 1.60 \\
Electricidad y agua & 0.16 & 0.14 & 0.14 \\
Comercio & 2.43 & 3.00 & 3.82 \\
Transporte & 0.68 & 0.79 & 1.06 \\
Servicios para empresas, & 2.52 & 3.43 & 4.45 \\
personas y el hogar & & & \\
Servicios sociales y comunales & 1.52 & 1.80 & 2.08 \\
Menos de 20 años & 0.49 & 0.38 & 0.61 \\
De 20 a 40 años & 8.16 & 9.17 & 11.30 \\
De 40 a 60 años & 3.46 & 4.75 & 6.54 \\
Más de 60 años & 0.29 & 0.40 & 0.53 \\
\hline
\end{tabular}

Nota: número de trabajadores asegurados al IMSS con salario que perciben más de un salario mínimo mensual. Fuente: elaboración propia con datos del IMSS.

Cuadro A3. México: ingreso mensual nominal (MXN)

\begin{tabular}{lccc}
\hline & 2005 & 2011 & 2017 \\
\hline Total & 7246 & 9798 & 13266 \\
$\begin{array}{l}\text { Tipo de contratación } \\
\text { Eventual (temporal) }\end{array}$ & 4105 & 6145 & 8590 \\
$\quad$ Permanente & 5175 & 6779 & 9082 \\
Grupo de edad & & & \\
Menos de 20 años & 3002 & 3810 & 5241 \\
De 20 a 40 años & 7448 & 9707 & 12942 \\
De 40 a 60 años & 8349 & 11073 & 14445 \\
Más de 60 años & 6541 & 8914 & 11194 \\
\hline
\end{tabular}

Nota: se reporta el ingreso promedio para trabajadores asegurados al IMSS con salario que perciben más de un salario mínimo mensual.

Fuente: elaboración propia con datos del IMSS. 


\section{Enrique Kato-Vidal}

Cuadro A4. Prueba de robustez: efectos de la legislación laboral en los salarios de trabajadores registrados a la seguridad social

\begin{tabular}{|c|c|c|c|c|c|c|}
\hline \multirow[b]{2}{*}{ Variable } & \multicolumn{2}{|c|}{ Total } & \multicolumn{2}{|c|}{ 20-39 años } & \multicolumn{2}{|c|}{ 40-59 años } \\
\hline & Coeficiente & $t$ & Coef. & $t$ & Coef. & $t$ \\
\hline Contrato permanente & 0.054 & 5.92 & 0.016 & 2.14 & 0.103 & 7.88 \\
\hline Año 2005 & -0.346 & -41.62 & -0.363 & -45.78 & -0.56 & -35.13 \\
\hline Año 2017 & 0.308 & 27.79 & 0.312 & 30.15 & 0.300 & 23.70 \\
\hline$(\text { Base })^{\star}(2005)$ & 0.073 & 7.44 & 0.111 & 12.24 & 0.058 & 4.94 \\
\hline$(\text { Base })^{\star}(2017)$ & -0.039 & -5.51 & -0.047 & -7.96 & -0.028 & -2.97 \\
\hline Hombre & 0.147 & 34.28 & 0.110 & 25.85 & 0.204 & 35.29 \\
\hline Extractivas & 0.395 & 11.05 & 0.411 & 10.38 & 0.368 & 10.99 \\
\hline Transformación & 0.198 & 10.66 & 0.195 & 11.42 & 0.196 & 8.74 \\
\hline Construcción & 0.234 & 10.74 & 0.230 & 10.91 & 0.235 & 9.58 \\
\hline Electricidad y agua & 0.879 & 39.53 & 0.835 & 39.83 & 0.928 & 36.70 \\
\hline Comercio & 0.239 & 9.68 & 0.240 & 10.00 & 0.240 & 8.77 \\
\hline Transporte & 0.356 & 14.02 & 0.356 & 15.09 & 0.365 & 12.51 \\
\hline Servicios para empresas, personas y el hogar & 0.284 & 11.48 & 0.306 & 12.93 & 0.278 & 10.07 \\
\hline Servicios sociales y comunales & 0.346 & 14.86 & 0.295 & 12.81 & 0.87 & 15.43 \\
\hline Entre 2 y 5 asegurados & 0.169 & 39.80 & 0.161 & 38.50 & 0.183 & 35.59 \\
\hline Entre 6 y 50 & 0.619 & 78.06 & 0.621 & 80.01 & 0.664 & 74.84 \\
\hline Entre 51 y 250 & 1.008 & 112.72 & 1.020 & 118.63 & 1.070 & 111.48 \\
\hline Entre 251 y 500 & 1.203 & 79.50 & 1.221 & 86.52 & 1.264 & 74.92 \\
\hline Entre 501 y 1000 & 1.313 & 112.31 & 1.335 & 124.00 & 1.372 & 105.64 \\
\hline Más de 1000 & 1.478 & 111.83 & 1.495 & 131.12 & 1.535 & 98.17 \\
\hline $\begin{array}{l}\text { Trabajadores en principales zonas } \\
\text { metropolitanas (CDMX, Estado de México, } \\
\text { Jalisco y Nuevo León) }\end{array}$ & 0.145 & 5.58 & 0.136 & 4.92 & 0.151 & 5.79 \\
\hline Observaciones & \multicolumn{2}{|c|}{10603996} & \multicolumn{2}{|c|}{5410595} & \multicolumn{2}{|c|}{4240018} \\
\hline Menos de 20 años & -0.657 & -48.60 & & & & \\
\hline De 40 a 60 años & 0.058 & 15.39 & & & & \\
\hline Más de 60 años & -0.152 & -15.35 & & & & \\
\hline
\end{tabular}

Notas: variable dependiente: logaritmo del salario base de cotización. Estimación de mínimos cuadrados ordinarios. El grupo de control fue el de trabajadores temporales (eventuales). Año de comparación (2011). Los coeficientes en negritas cuantifican el efecto de la legislación laboral. Las estimaciones incluyen un término constante que no se reporta. Categorías de referencia: Sexo (mujer), Sector (agropecuario). Tamaño de registro patronal (un asegurado). Trabajadores en principales zonas metropolitanas (resto del país). Edad (20-39 años).

Fuente: elaboración propia con datos del IMSS. 


\section{BIBLIOGRAFÍA}

Abadie, A. (2008), "Difference-in-difference estimators", The New Palgrave Dictionary of Economics, vol. 1-8, DOI <https://doi.org/10.1057/978-1349-95121-5_2593-1>

Banco de México (2013), "Estimación del efecto de la Reforma Laboral sobre el crecimiento del PIв potencial y el empleo formal", Extracto del Informe sobre la Inflación octubre-diciembre 2012, Recuadro 2, febrero.

Casar, J. I. (2019), "Jaime Ros Bosch, economista", El Trimestre Económico, vol. 86, núm. 344, DOI <http://dx.doi.org/10.20430/ete.v86i344.978>

Cervini-Plá, M., Ramos, X. y Silva, J. I. (2014), "Wage effects of non-wage labour costs", European Economic Review, vol. 72, DOI <https://doi. org/10.1016/j.euroecorev.2014.09.005>

Davia, M. A. y Hernanz, V. (2004), "Temporary employment and segmentation in the spanish labour market: An empirical analysis through the study of wage differentials", Spanish Economic Review, vol. 6, núm. 4, DOI $<10.1007 /$ s10108-004-0086-2>

Gandolfi, D., Halliday, T. y Robertson, R. (2017), "Trade, FDI, migration, and the place premium: Mexico and the United States", Review of World Economics, vol. 153, núm. 1, DOI <https://doi.org/10.1007/s10290-0160260-2>

Gialis, S., Tsampra, M. y Leontidou, L. (2017), "Atypical employment in crisis-hit greek regions: Local production structures, flexibilization and labour market re/deregulation", Economic and Industrial Democracy, vol. 38, núm. 4, DOI <https://doi.org/10.1177/0143831X15586815>

Hernández Laos, E. (2013), "Legislación laboral, sector informal y productividad multifactorial en México", Economía UNAM, vol. 10, núm. 28.

Ibarra, C. A. y Ros, J. (2019), "The decline of the labor income share in Mexico, 1990-2015", World Development, vol. 122, DoI <https://doi. org/10.1016/j.worlddev.2019.06.014>

Imbens, G. W. y Wooldridge, J. M. (2009), "Recent developments in the econometrics of program evaluation", Journal of economic literature, vol. 47, núm. 1, DoI <10.1257/jel.47.1.5>

Instituto Nacional de Estadística y Geografía (INEGI) (2007), Cómo se hace la ENOE: métodos y procedimientos, México, INEGI.

Lewis, W. A. (1954), "Economic development with unlimited supplies of labour", The Manchester School, vol. 22, núm. 2, DOI <https://doi. org/10.1111/j.1467-9957.1954.tb00021.x> 
Márquez, C. y Ros, J. (1990), "Segmentación del mercado de trabajo y desarrollo económico en México", El Trimestre Económico, vol. 57, núm. 226 (2). Disponible en <www.jstor.org/stable/23397538>

Perrotini, I. (2014), "Jaime Ros Bosch. Algunas tesis equivocadas sobre el estancamiento económico de México", El Trimestre Económico, vol. 81, núm. 324, DOI <http://dx.doi.org/10.20430/ete.v81i324.138>

Ros, J. (2004), La teoría del desarrollo y la economía del crecimiento, México, Fondo de Cultura Económica.

(2013a), "Introducción a repensar el desarrollo económico, el crecimiento y las instituciones", Economía UNAM, vol. 10, núm. 30. Disponible en <http://www.revistas.unam.mx/index.php/ecu/article/view/45114> (2013b), Rethinking economic development, growth and institutions, Oxford, Oxford University Press (2013c), Algunas tesis equivocadas sobre el estancamiento económico de México, México, El Colegio de México-unam.

(2015), ¿Cómo salir de la trampa del lento crecimiento y alta desigualdad?, México, El Colegio de México-unam.

(2019), "Dinámica empresarial disfuncional y productividad estancada: una reseña del nuevo libro de Santiago Levy", Economía UNAM, vol. 16, núm. 46. 